\title{
Factors Associated with Pelvic Floor Muscle Strength in Women with Pelvic Floor Dysfunction Assessed by the Brink Scale
}

Sirirat Sarit-apirak, R.N., M.Sc. , Jittima Manonai, M.D., M.H.M. ${ }^{2}$, Umaporn Udomsubpayakul, M.Sc. ${ }^{3}$

'Department of Nursing, Somdech Phra Debaratana Medical Center, ${ }^{2}$ Department of Obstetrics \& Gynaecology,

${ }^{3}$ Section for Clinical Epidemiology and Biostatistics, Faculty of Medicine Ramathibodi Hospital, Mahidol University,

Ratchathewi, Bangkok 10400, Thailand.

Received 5 July 2019 • Revised 23 August 2019 • Accepted 26 August 2019 • Published online 29 October 2019

\begin{abstract}
:
Objective: (1) to examine the pelvic floor muscle (PFM) function using the Brink scale and (2) to investigate the correlation between potential factors and PFM function.

Material and Methods: From January 2011 and December 2014, women with at least one pelvic floor symptom attending the urogynecology clinic were included in a medical record review. Demographic and pelvic floor symptoms were assessed. The Brink scoring system was used to assess the PFM function. The association between factors and Brink scale scores was measured using Pearson's Correlation Coefficient.

Results: Five hundred and seventy-nine women with a mean age of $64.40 \pm 10.11$ years were included in the analysis. Forty-seven women (8.1\%) were unable to contract their pelvic floor muscle at all, while 55 (9.5\%) could both powerfully and properly. The mean Brink scale score was $7.82 \pm 2.56$. Elderly women had a significantly lower score than younger women (mean scores of $7.56 \pm 2.60$ and $8.08 \pm 2.50$, respectively) with the mean score in nulliparous and parous women being $8.66 \pm 2.63$ and $7.76 \pm 2.55$, respectively ( $p$-value=0.046). A negatively weak correlation was found among those with higher total scores and advancing age (correlation $(r)=-0.106)$, advanced anterior $(r=-0.095)$ and apical compartment $(r=-0.105)$ prolapse $(p-$ value $<0.05)$.
\end{abstract}

The abstract was presented at the International Urogynecological Association $43^{\text {rd }}$ Annual Meeting, Vienna, Austria, June 27-30, 2018. Contact: Prof. Jittima Manonai, M.D., M.H.M.

Department of Obstetrics \& Gynaecology, Faculty of Medicine Ramathibodi

Hospital, Mahidol University, Ratchathewi, Bangkok 10400, Thailand.

E-mail: jittima.man@mahidol.ac.th

This is an open access article under the CC BY-NC-ND license

(http://www.jhsmr.org/index.php/jhsmr/about/editorialPolicies\#openAccessPolicy). 
Conclusion: Almost all the women with pelvic floor dysfunction had compromised pelvic floor function. Important factors affecting PFM strength are age, parity, and history of hysterectomy. Increasing age, higher stage of anterior and apical compartment prolapse were negatively correlated with PFM function.

Keywords: Brink scale, pelvic floor dysfunction, pelvic floor muscle strength, pelvic organ prolapse

\section{Introduction}

Abnormalities of pelvic floor muscles (PFM) lead to pelvic floor dysfunction, which can be clinically categorized into lower urinary symptoms, urinary tract infection, pelvic organ prolapse, anorectal dysfunction, sexual problems, and lower urinary tract pain and/or other pelvic pain. ${ }^{1}$ These conditions are generally prevalent and cause significant morbidity in terms of well-being and quality of life in women. If diagnosed at an early stage, certain conservative treatment, such as behavioral changes, pelvic floor muscle therapy, medications, and pessaries may relieve symptoms as well as prevent the need for invasive treatment or future surgery. ${ }^{2,3}$

Pelvic floor muscles apply to the muscular layers of the pelvic floor and are crucial in pelvic organ support and continence process. In the course of various chapters of a woman's lifetime, including pregnancy, childbirth, aging and menopause, striated muscles of the pelvic floor become damaged, or if its innervation is impaired, the muscle function will be negatively affected. Factors like these, may diminish the durability of the PFM and result in involuntary loss of bladder or rectal contents in addition to pelvic organ prolapse. Pelvic floor muscle training (PFMT) is endorsed as a first-line conservative treatment for pelvic organ prolapse and urinary incontinence. ${ }^{4} \mathrm{~A}$ voluntary contraction of the pelvic floor muscles can be assessed using finger(s) as part of assessment to gather information about the muscles and surrounding area., ${ }^{5,6}$ Currently, the Brink scale is the most commonly used method for digital assessment of PFM function, as it can be performed by any trained physician, physiotherapist or nurse. This method can measure strength and endurance of the pelvic floor muscles. Moreover, a previous study demonstrates a strong correlation between perineometer evaluations and Brink scores in pelvic floor muscle strength assessment. ${ }^{7}$ Investigating PFM function along with associated factors may guide the selection for the specific training protocols or specific techniques, for women with different pelvic floor symptoms or different risk factors. To date, limited information is available on the function of the pelvic floor muscles or the associated factors for women with and without pelvic floor dysfunction. Hence, the objectives of this study were (1) to examine the pelvic floor muscle function using the Brink scale and (2) to investigate the correlation between women's characteristics, pelvic floor symptoms, stage of pelvic organ prolapse and PFM function in women with pelvic floor symptoms.

\section{Material and Methods}

\section{Participants}

After institutional review board approval (protocol number ID 04-59-47) was obtained, a retrospective chart review of consecutive women attending a urogynecology clinic of a university hospital, from January 2011 to December 2014 was conducted. Exclusion criteria included pregnancy, neurological disorders, women without pelvic floor symptoms and those with incomplete data. Demographic data on age, body mass index, parity, menopausal status and history of hysterectomy were retrieved from the electronic medical records system. 
Pelvic floor dysfunction and pelvic organ prolapse

The Pelvic Floor Bother Questionnaire (PFBQ) was used to evaluate pelvic floor symptoms. ${ }^{8}$ This is a self- administered questionnaire that includes nine symptoms: stress urinary incontinence, urinary urgency and frequency, urgency urinary incontinence, dysuria, pelvic organ prolapse, obstructed defecation, fecal incontinence and dyspareunia. A Thai version of the PFBQ was translated and validated for cross-cultural research. The Thai version was tested as well as used in a previous study with a reliability coefficient of 0.95 . $^{9}$ Pelvic organ prolapse was evaluated according to the pelvic organ prolapse quantification (POP-Q) system ${ }^{10}$ by urogynecologists. Six defined points on the anterior vaginal wall, the superior vagina, and the posterior vaginal wall were measured and located with reference to the hymeneal ring. POP stages I-IV are assigned as stated by the most distal portion of the prolapse when the maximum descent has been attained.

\section{Pelvic floor muscle function measurement}

Assessment of PFM was performed by a urogynecologist or a specialized nurse according to the Brink scale scoring protocol manual. ${ }^{11}$ Intra-rater and inter-rater reliability were tested previously and the good levels were demonstrated (0.865-0.907 and 0.698-0.882, respectively). Women are positioned on their back with their hips and knees flexed, then the examiner's index finger is inserted, up to approximately 1.5 to 2.5 inches, into the vaginal canal. The woman is then told to both relax and then squeeze her pelvic floor muscles, whilst lifting it inside, without contracting her abdomen, hip and thigh muscles. In the evaluation of three PFM function variables the Brink scale was used. These comprised of vaginal pressure, length of contraction, and vertical movement of the examiner's fingers. Each muscle contraction variable was rated on a 4-point ordinal scale from 1 to 4 .
The vaginal pressure was scored from no pressure at all (1) to strong squeeze with full circumference of fingers compressed (4). The length of contraction was quantified from 0 second (1) to longer than 3 seconds (4). The vertical displacement was defined from no movement (1) to the whole finger moves anteriorly and is pulled in (4). Then, total scores based on the sum of three items were obtained, with a possible range of 3 (minimum) to 12 (maximum). The higher the score reflects stronger PFM function. The procedure was repeated three times, with the median value of the total measurements used.

\section{Statistical analyses}

Both descriptive and analytical analyses were conducted using a Statistical Package for the Social Science (SPSS version 18.0). The Kolmogorov-Smirnov test was used to determine whether the data were distributed normally. Data were presented as $\mathrm{n}(\%)$ and mean \pm standard deviation, and median (interquartile) were used as appropriate to describe demographic variables along with the strength and endurance of the pelvic floor muscle of women. Independent sample t-test or Mann-Whitney $U$ test were conducted to examine the differences of Brink scale scores between demographic data, pelvic floor symptoms, stage. One-way analysis of variance or Kruskal-Wallis test were conducted to examine the differences of Brink scale scores between Pelvic organ prolapse stages. Pearson's product-moment correlation coefficient was used to measure the correlation between Brink scale scores, potential variables and PFM function. A $p$-value $<0.05$ was considered statistically significant.

\section{Results}

From January 2011 to December 2014, 747 women attended the urogynecology clinic for the first time. Of these, 168 women were excluded as stated by the exclusion criteria. Included in the analysis were 579 women with complete information. Among these women, the mean 
age was $64.40 \pm 10.11$ years, and the mean body mass index was $25.60 \pm 3.89 \mathrm{~kg} / \mathrm{m}^{2}$. Those that were parous numbered 544 (93.9\%) 50 women (10.2\%) had undergone a hysterectomy; additionally 479 (82.7\%) individuals were postmenopausal (Table 1). Urgency urinary incontinence and stress urinary incontinence were reported in 253 women $(43.7 \%)$ and 275 women $(47.5 \%)$ in the past month.

Table 2 shows the scores for the Brink scale scores in women with pelvic floor dysfunction in dimensions of (1) squeeze pressure, (2) length of contraction, and (3) vertical movement of the examiner's fingers. The mean total Brink scale score was $7.82 \pm 2.56$ with a median of $8(6,10)$. For those women attending a urogynecology clinic with at least one of the pelvic floor symptoms, $47(8.1 \%)$ were unable to conduct pelvic floor

Table 1 Demographic variables and prevalence of pelvic floor dysfunction $(n=579)$

\begin{tabular}{|c|c|}
\hline Characteristics/Pelvic floor dysfunction & $\begin{array}{l}\text { Mean士S.D. } \\
\text { Median } \\
(\min , \max ) \\
\text { Number }(\%)\end{array}$ \\
\hline Age (years); mean \pm S.D. & $64.40 \pm 10.11$ \\
\hline Body mass index $\left(\mathrm{kg} / \mathrm{m}^{2}\right)$; mean \pm S.D. & $25.60 \pm 3.89$ \\
\hline Parity; median (min, max) & $3.00(1-10)$ \\
\hline Parous; number (\%) & $544(94.0)$ \\
\hline Postmenopause status; number (\%) & $479(82.7)$ \\
\hline Hysterectomy; number (\%) & $59(10.2)$ \\
\hline \multicolumn{2}{|l|}{ Pelvic floor symptoms; number (\%) } \\
\hline Stress urinary incontinence & $275(47.5)$ \\
\hline Urgency urinary incontinence & $253(43.7)$ \\
\hline Defecatory difficulty & $170(29.4)$ \\
\hline \multicolumn{2}{|c|}{ Pelvic organ prolapse (overall stage); number (\%) } \\
\hline I & $81(14.0)$ \\
\hline II & $221(38.2)$ \\
\hline III & $186(32.1)$ \\
\hline IV & $91(15.7)$ \\
\hline
\end{tabular}

S.D.=standard deviation, $\mathrm{kg}=$ kilogram, $\mathrm{m}=$ meter muscle exercises at all (total score of 3), whereas 55 $(9.5 \%)$ did powerfully and properly (total score of 12 ). Regarding Brink scale quartile among women who were able to contract their PFM; 151 (26.1\%), 171 (29.5\%), and $154(26.6 \%)$ were in the lower, middle and upper quartiles, respectively.

The current study revealed that age, parity, and history of hysterectomy were factors that significantly affected the PFM function whereas body mass index, route of delivery, ovarian function, or pelvic floor symptoms did not (Table 3 ). Elderly women (age $\geq 65$ years old) had a significantly lower score than younger women, with the mean scores being $7.56 \pm 2.60$ and $8.08 \pm 2.50$, respectively. The mean Brink scale score in nulliparous and parous women were $8.66 \pm 2.63$ and $7.76 \pm 2.55$, respectively $(p-$ value $=0.046)$.

Table 2 Brink scale scores in women with pelvic floor dysfunction $(n=579)$

\begin{tabular}{|c|c|c|}
\hline Dimensions & Number & Percent \\
\hline \multicolumn{3}{|l|}{ Squeeze pressure; mean $2.74 \pm 0.88$} \\
\hline $1=$ no response & 53 & 9.2 \\
\hline $2=$ weak squeeze & 160 & 27.6 \\
\hline $3=$ moderate squeeze & 252 & 43.5 \\
\hline $4=$ strong squeeze & 114 & 19.7 \\
\hline \multicolumn{3}{|l|}{ Muscle contraction duration; mean } \\
\hline \multicolumn{3}{|l|}{$2.72 \pm 0.93$} \\
\hline $1=$ none & 63 & 10.9 \\
\hline $2=<1$ second & 165 & 28.5 \\
\hline $3=1-3$ seconds & 222 & 38.3 \\
\hline $4=>3$ seconds & 129 & 22.3 \\
\hline \multicolumn{3}{|l|}{ Displacement of vertical plane; mean } \\
\hline \multicolumn{3}{|l|}{$2.37 \pm 0.94$} \\
\hline $1=$ none & 111 & 20.2 \\
\hline $2=$ finger base moves anteriorly & 207 & 35.8 \\
\hline $\begin{array}{l}3=\text { whole length of finger moves } \\
\text { anteriorly }\end{array}$ & 181 & 31.3 \\
\hline $\begin{array}{l}4=\text { whole finger moves anteriorly, } \\
\text { are gripped and pulled in }\end{array}$ & 74 & 12.8 \\
\hline
\end{tabular}


Table 3 Comparisons of mean \pm S.D. of Brink scale scores among different characteristics and pelvic floor dysfunction

\begin{tabular}{|c|c|c|c|}
\hline \multirow[t]{2}{*}{ Characteristics } & \multicolumn{2}{|c|}{$\begin{array}{l}\text { Brink scale } \\
\text { scores }\end{array}$} & \multirow[t]{2}{*}{$\mathrm{p}$-value } \\
\hline & Mean & S.D. & \\
\hline Age (years) & & & 0.014 \\
\hline$<65$ & 8.08 & 2.50 & \\
\hline$\geq 65$ & 7.56 & 2.60 & \\
\hline Body mass index & & & 0.867 \\
\hline$<25.0$ & 7.84 & 2.53 & \\
\hline$\geq 25.0$ & 7.80 & 2.59 & \\
\hline Parity & & & 0.046 \\
\hline Nulliparous & 8.66 & 2.63 & \\
\hline Parous & 7.76 & 2.55 & \\
\hline Route of delivery & & & 0.893 \\
\hline Vaginal delivery & 7.76 & 2.55 & \\
\hline Caesarean section & 7.84 & 2.67 & \\
\hline Hysterectomy & & & 0.022 \\
\hline Yes & 8.54 & 2.65 & \\
\hline No & 7.74 & 2.54 & \\
\hline Pelvic floor symptoms & & & \\
\hline Stress urinary incontinence & & & 0.519 \\
\hline Present & 7.89 & 2.51 & \\
\hline Absent & 7.75 & 2.61 & \\
\hline Urgency urinary incontinence & & & 0.358 \\
\hline Present & 7.71 & 2.50 & \\
\hline Absent & 7.90 & 2.61 & \\
\hline Defecatory difficulty & & & 0.623 \\
\hline Present & 7.90 & 2.62 & \\
\hline Absent & 7.78 & 2.54 & \\
\hline Pelvic organ prolapse stage* & & & 0.451 \\
\hline Stage I & 8.10 & 2.57 & \\
\hline Stage II & 7.92 & 2.52 & \\
\hline Stage III & 7.71 & 2.56 & \\
\hline Stage IV & 7.55 & 2.65 & \\
\hline
\end{tabular}

t-test

${ }^{*}$ Analysis of variance (ANOVA)

S.D.=standard deviation, $\mathrm{p}-$ value $=$ calculated probability

Interrelationship between demographic data, POP-Q results (anterior, apical and posterior compartment) and the total Brink scale scores are shown in Table 4.
Correlation analysis determined a significant negative relationship between the higher total Brink scale scores and advancing age $(r=-0.106)$, advanced anterior or point Ba ( $r=-0.095)$ and apical compartment or point C $(r=$ -0.105) prolapse ( $p$-value<0.05).

Table 4 Correlations between characteristic and Pelvic Organ Prolapse Quantification findings, and the total Brink scale scores

\begin{tabular}{lcl}
\hline Factors & Correlation $(\mathbf{r})$ & $\mathbf{p}$-value \\
\hline Age (years) & -0.106 & $0.010^{*}$ \\
Body mass index $\left(\mathrm{kg} / \mathrm{m}^{2}\right)$ & 0.005 & 0.912 \\
Parity & -0.062 & 0.139 \\
Anterior compartment $(\mathrm{Ba})$ & -0.103 & $0.023^{*}$ \\
Apical compartment $(\mathrm{C})$ & -0.105 & $0.011^{*}$ \\
Posterior compartment $(\mathrm{Bp})$ & -0.066 & 0.115 \\
Genital hiatus $(\mathrm{cm})$ & -0.011 & 0.796 \\
Perineal body $(\mathrm{cm})$ & -0.004 & 0.093 \\
\hline
\end{tabular}

Pearson correlation

$\mathrm{p}$-value=calculated probability, $r=$ correlation, $\mathrm{kg}=$ kilogram,

$\mathrm{m}=$ meter, $\mathrm{Ba}=$ point $\mathrm{B}$ on anterior vaginal wall, $\mathrm{C}=$ cervix or cuff,

$\mathrm{Bp}=$ point $\mathrm{B}$ on posterior vaginal wall, $\mathrm{cm}=$ centimeter

${ }^{*} p-$ value $<0.05$

\section{Discussion}

A correct contraction, meaning a squeeze around the vaginal opening and an elevation of the pelvic floor define strong pelvic floor muscle function. ${ }^{12}$ Remarkably, most women with pelvic floor symptoms in this study could contract their pelvic floor muscles; as well as which about $10.0 \%$ achieved the maximum score. This might be explained by the women's knowledge coupled with awareness of pelvic floor muscle exercises during their previous encounter with health care providers, before being referred to our institute. Both mean and median Brink scale scores reported in this present study are comparable with previous 
findings in women with pelvic organ prolapse ${ }^{13}$, stress urinary incontinence ${ }^{14}$, and postpartum anal incontinence. ${ }^{15}$

Regarding the associated factors, this study demonstrates a possible important relationship between compromised pelvic floor muscle function with advancing age, parity, history of hysterectomy, anterior vaginal wall prolapse and apical compartment prolapse. These observations are consistent with findings from previous studies and an etiologic theory of pelvic floor dysfunction. ${ }^{12-14}$ Basically, a $40.0 \%$ reduction in the force that the pelvic floor muscles can generate during a pelvic muscle contraction is associated with pelvic floor muscle injury. ${ }^{16}$ It can be implied that conditions that cause pelvic floor muscle damage; for example: parity, repeated heavy lifting, obesity, being constipated or degenerative changes, like; aging and menopause lead to pelvic floor muscles weakening during contraction.

The pelvic floor muscles hold the pelvic floor closed, providing lifting and closing forces to prevent the vaginal walls descending through the levator hiatus. ${ }^{17}$ Loss of pelvic organ support as a consequence of pregnancy, vaginal birth, menopause and aging is found to be one of the risk factors for pelvic organ prolapse. The impaired PFM function may compromise pelvic organ support and contribute to the development of prolapse. Although correlations were found between weaker PFM function and advanced prolapse of anterior and apical compartments, the PFM were not stronger in women with non-advanced pelvic organ prolapse compared to the advanced stage group. It is possible that the Brink method may not be sufficiently applicable to determine comprehensive pelvic floor muscle function. This may be in part due to; connective tissue failure occuring in the attachments of the vaginal wall to the pelvic sidewall, rather than failure of pelvic floor muscle. ${ }^{18}$ Interestingly, there was no correlation between PFM function and posterior wall prolapse, which is mainly supported by the puborectal portion of the levator ani muscle. This might be explained by the findings from a previous report, which found major defects and loss of muscle bulk only in the pubovisceral portion but not in the puborectal portion of the muscle from magnetic resonance imaging in women with pelvic organ prolapse. ${ }^{19}$

An inappropriate exercise-training program may decrease adherence and outcomes. Before starting any program, pelvic floor muscle strength along with endurance should be systematically evaluated and associated factors for PFM function should be cautiously considered. An initial evaluation based on pelvic floor muscle function would help to define the best individualized pelvic floor muscle training. Thus, a more specific muscle training regimen adjoined with an individual, particular technique; for example, cushion or abdominal muscle-assisting techniques or biofeedback, could be properly designed for each woman in order to achieve the greatest benefit. In addition, contemplating all known associated factors for pelvic floor function would be helpful to determine a PFMT prognosis and indicate any further treatment as required.

This study consists of a number of strengths such as the systematic evaluation of pelvic floor symptoms using a simple, validated questionnaire and that both stage and compartment of pelvic organ prolapse using $P O P-Q$ was conducted. The Brink scoring system, a reliable clinical tool of PFM testing was also used. Even though the Brink scale is a subjective method to assess pelvic floor muscle strength, total Brink scores utilizing three subscales, showed fair to good correlations with the results obtained from the perineometer in the previous study. ${ }^{7}$ Additionally, all assessors went through a standardized process, in order to ensure that they all followed the same assessment procedure, so as to make reliable decisions.

However, the study produced a variety of weaknesses that require attention. This was a retrospective study conducted in a subspecialized, academic setting; hence selection bias may have been inevitable. As a result, 
the extension of research findings and conclusions to the general population may be compromised. Another weakness in this study is that the PFM function examiners were not blinded to the results of the POP-Q results before examining the women. In addition, activities of abdominal muscles and synergist muscles around the hip joint especially adductor magnus and gluteus maximus were not evaluated. It is hypothesized that these muscles affect the performance of the pelvic floor muscle. ${ }^{20,21}$ Furthermore, a previous study in menopausal study reported that PFM function depends on various lower limb positions, and that the supine position is recommended for diagnosis of weakened PFM. ${ }^{22}$ Therefore, assessing PFM and other synergists functions simultaneously in a supine position should be performed in further studies.

\section{Conclusion}

Among women attending the urogynecology clinic with pelvic floor dysfunction, almost all of them had compromised pelvic floor function. PFM strength and endurance evaluated with the Brink scale are affected by important factors; these-being: age, parity, and history of hysterctomy. Significant factors affecting PFM strength and endurance evaluated with the Brink scale are age and parity. Advancing age, higher stage of anterior and apical prolapse were negatively correlated with PFM function.

\section{Conflict of interest}

None

\section{References}

1. Haylen BT, de Ridder D, Freeman RM, Swift SE, Berghmans B,

Lee $\mathrm{J}$, et al. An International Urogynecological Association (IUGA)/nternational Continence Society (ICS) joint report on the terminology for female pelvic floor dysfunction. Int Urogynecol J 2010;21:5-26.

2. Arnouk A, De E, Rehfuss A, Cappadocia C, Dickson S, Lian F. Physical, complementary, and alternative Medicine in the treatment of pelvic floor disorders. Curr Urol Rep 2017;18:47.
3. Angelini K. Pelvic floor muscle training to manage overactive bladder and urinary incontinence. Nurs Womens Health 2017; 21:51-7.

4. Dumoulin C, Cacciari LP, Hay-Smith EJC. Pelvic floor muscle training versus no treatment, or inactive control treatments, for urinary incontinence in women. Cochrane Database Syst Rev 2018. doi: 10.1002/14651858.CD005654.pub4.

5. Bo K, Frawley HC, Haylen BT, Abramov Y, Almeida FG, Berghmans B, et al. An International Urogynecological Association (IUGA) /nternational Continence Society (ICS) joint report on the terminology for the conservative and nonpharmacological management of female pelvic floor dysfunction. Int Urogynecol J 2017; 28:191-213.

6. Messelink B, Benson T, Berghmans B, Bo K, Corcos J, Fowler C, et al. Standardization of terminology of pelvic floor muscle function and dysfunction: report from the pelvic floor clinical assessment group of the International Continence Society. Neurourol Urodyn 2005;24:374-80.

7. Hundley AF, Wu JM, Visco AG. A comparison of perineometer to brink score for assessment of pelvic floor muscle strength. Am J Obstet Gynecol 2005;192:1583-91.

8. Peterson TV, Karp DR, Aguilar VC, Davila GW. Validation of a global pelvic floor symptom bother questionnaire. Int Urogynecol J 2010;21:1129-35.

9. Manonai J, Wattanayingcharoenchai R. Relationship between pelvic floor symptoms and POP-Q measurements. Neurourol Urodyn 2016;35:724-7.

10. Bump RC, Mattiasson A, Bo K, Brubaker LP, DeLancey JO, Klarskov $\mathrm{P}$, et al. The standardization of terminology of female pelvic organ prolapse and pelvic floor dysfunction. Am $\mathrm{J}$ Obstet Gynecol 1996;175:10-7.

11. Brink CA, Sampselle CM, Wells TJ, Diokno AC, Gillis GL. A digital test for pelvic floor muscle strength in older women with urinary incontinence. Nurs Res 1989;38:196-9.

12. Bo K, Sherburn M. Evaluation of female pelvic-muscle function and strength. Phys Ther 2005;85:269-82.

13. Borello-France DF, Handa VL, Brown MB, Goode P, Kreder K, Scheufele LL, et al. Pelvic-floor muscle function in women with pelvic organ prolapse. Phys Ther 2007;87:399-407.

14. FitzGerald MP, Burgio KL, Borello-France DF, Menefee SA, Schaffer J, Kraus S, et al. Pelvic-floor strength in women with incontinence as assessed by the brink scale. Phys Ther 2007:87:1316-24. 
15. King VG, Boyles SH, Worstell TR, Zia J, Clark AL, Gregory WT. Using the Brink score to predict postpartum anal incontinence. Am J Obstet Gynecol 2010;203:486.e1-5.

16. DeLancey JO, Morgan DM, Fenner DE, Kearney R, Guire K, Miller JM, et al. Comparison of levator ani muscle defects and function in women with and without pelvic organ prolapse. Obstet Gynecol 2007;109:295-30.

17. Delancey JO. Surgery for cystocele III: do all cystoceles involve apical descent? Observations on cause and effect. Int Urogynecol J 2012;23:665-7.

18. Delancey JO. What's new in the functional anatomy of pelvic organ prolapse? Curr Opin Obstet Gynecol 2016;28:420-9.

19. DeLancey JO, Sørensen HC, Lewicky-Gaupp C, Smith TM. Comparison of the puborectal muscle on MRI in women with POP and levator ani defects with those with normal support and no defect. Int Urogynecol J 2012;23:73-7.
20. Bo K, Morkved S, Frawley H, Sherburn M. Evidence for benefit of transversus abdominis training alone or in combination with pelvic floor muscle training to treat female urinary incontinence: a systematic review. Neurourol Urodyn 2009; 28:368-73.

21. Soljanik I, Janssen U, May F, Fritsch H, Stief CG, Weissenbacher $E R$, et al. Functional interactions between the fossa ischioanalis, levator ani and gluteus maximus muscles of the female pelvic floor: a prospective study in nulliparous women. Arch Gynecol Obstet 2012;286:931-8.

22. Halski T, Ptaszkowski K, Stupska L, Dymarek R, PaprockaBorowicz M. Relationship between lower limb position and pelvic floor muscle surface electromyography activity in menopausal women: a prospective observational study. Clin Intervent Aging 2017;12:75-83. 